\title{
A Linda-based Platform for the Parallel Execution of Out-place Model Transformations
}

\author{
Loli Burgueño ${ }^{1}$, Manuel Wimmer ${ }^{2}$, and Antonio Vallecillo ${ }^{1}$ \\ 1 Universidad de Málaga, Spain. \{loli, av\} @ lcc. uma.es \\ 2 Christian Doppler Laboratory for Model-Integrated Smart Production, TU Wien, Austria. \\ wimmerabig.tuwien.ac.at
}

\begin{abstract}
.
Context: The performance and scalability of model transformations is gaining interest as industry is progressively adopting model-driven techniques and multicore computers are becoming commonplace. However, existing model transformation engines are mostly based on sequential and in-memory execution strategies, and thus their capabilities to transform large models in parallel and distributed environments are limited.

Objective: This paper presents a solution that provides concurrency and distribution to model transformations.

Method: Inspired by the concepts and principles of the Linda coordination language, and the use of data parallelism to achieve parallelization, a novel Javabased execution platform is introduced. It offers a set of core features for the parallel execution of out-place transformations that can be used as a target for high-level transformation language compilers.

Results: Significant gains in performance and scalability of this platform are reported with regard to existing model transformation solutions. These results are demonstrated by running a model transformation test suite, and by its comparison against several state-of-the-art model transformation engines.

Conclusion: Our Linda-based approach to the concurrent execution of model transformations can serve as a platform for their scalable and efficient implementation in parallel and distributed environments.
\end{abstract}

Keywords: Model Transformation, Performance, Scalability, Parallelization

\section{Reference}

1. Burgueño, L., Wimmer, M., Vallecillo, A.: A Linda-based platform for the parallel execution of out-place model transformations. Information and Software Technology 79, 17 - 35 (2016), (DOI: 10.1016/j.infsof.2016.06.001) 